\title{
Une infiltration péri-rénale gauche
}

A left peri-renal infiltration

E. Masson ${ }^{a}$, U.-A. Scemama ${ }^{b}$, M.-A. Lignères ${ }^{b}$, A. Benyamine ${ }^{a}$, P. Rossi ${ }^{a}$, K. Chaumoitre $^{b}$, B. Granel ${ }^{a,{ }^{*}}$

a Service de médecine interne, hôpital Nord, chemin des Bourrely, 13915 Marseille cedex 20, France

${ }^{b}$ Service d'imagerie médicale, hôpital Nord, chemin des Bourrely, 13915 Marseille cedex 20, France Auteur correspondant. Service de médecine interne, hôpital Nord, Assistance Publique Hôpitaux de Marseille, 13915 Marseille, France. Adresse e-mail : bgranel@ap-hm.fr (B. Granel).

\section{L'histoire}

Un homme, âgé de 81 ans, vivant en institution, était hospitalisé en urgence pour une douleur abdominale associée à une constipation (pas de selles depuis 4 jours). Ses antécédents notables étaient une hypertension artérielle, un accident vasculaire cérébral ischémique sans séquelle, une fibrillation auriculaire et des troubles cognitifs. Ses traitements comprenaient : bisoprolol, lercanidipine, fluindione et paroxétine. À l'examen, les constantes hémodynamiques étaient conservées, sans fièvre. L'abdomen était tendu avec une matité sus pubienne. On notait aussi un important météorisme et une défense du flanc gauche. Le toucher rectal confirmait le fécalome. II n'y avait pas de signes de décompensation cardiaque. La biologie objectivait une insuffisance rénale aiguë sévère (clairance de la créatinine : $8 \mathrm{~mL} / \mathrm{min} / 1,73 \mathrm{~m} 2$ selon Chronic Kidney Disease Epidemiology Collaboration) et un syndrome inflammatoire (CRP $>350 \mathrm{mg} / \mathrm{L}$ ) avec hyperleucocytose à polynucléaires neutrophiles, sans anémie. L'INR était à 6 . La conduite thérapeutique immédiate consistait à la mise en place d'une sonde urinaire permettant l'évacuation de 4 litres d'urines hématuriques, une hydratation intraveineuse pour prévenir le syndrome de levé d'obstacle et la réalisation de lavements rectaux, ramenant des selles. Un traitement antibiotique par céphalosporine de troisième génération était débuté dans l'hypothèse d'une porte d'entrée infectieuse urinaire ou digestive. L'examen cytobactériologique urinaire confirmait par la suite une infection à Klebsiella pneumoniae sensible à la ceftriaxone. Les hémocultures restaient stériles. Aux urgences, devant l'INR élevé, l'insuffisance rénale aiguë et les urines hématuriques, la fluindione était suspendue et $2 \mathrm{mg}$ de vitamine $\mathrm{K}$ était administrée. Le premier scannerabdominal sans injection retrouvait un épanchement périrénal et pararénal postérieur et antérieur gauche, de densité liquidienne avec sédiment dense, sans dilatation significative des cavités excrétrices urinaires. L'uroscanner était secondairement réalisé après normalisation de la fonction rénale (Fig. 1).

2. Le diagnostic

Un urinome.

3. Les commentaires

L'urinome est une rare condition correspondant à une extravasation d'urine encapsulée dont l'origine, traumatique ou spontanée, est toujours liée à un facteur obstructif. Sa principale cause est l'obstacle urétéral sur lithiase [1]. D'autres causes d'urinome sont rapportées dans la littérature : obstruction sur néoplasie rectale [2], traumatique sur choc abdominal [3], manipulation iatrogène lors d'une endoscopie urétérale [4], et sur syndrome de la jonction pyélo-urétérale, avec un diagnostic prénatal possible pour cette dernière cause [5]. L'originalité de notre observation est la cause de l'obstruction secondaire à l'important globe vésical surfécalome. La rupture des voies excrétrices permettrait de lever la surpression sur le parenchyme (effet " pop-off » ou " soupape ») et protégerait ainsi le rein. Ceci est bien décrit dans les cas d'urinomes sur obstacle sous vésical avec constitution d'ascite urinaire. Les complications de l'urinome sont d'ordre mécanique ou infectieux. 
Le diagnostic est réalisé par scanner (images réalisées au moins 6 minutes après l'injection du produit de contraste), l'échographie étant moins performante. Le traitement repose essentiellement sur la levée de l'obstacle ( $s$ 'il existe) et la protection de la voie excrétrice lésée (dérivation des urines et mise en place d'une sonde JJ par voie percutanée ou naturelle). Le drainage percutané de l'urinome est parfois associé si son volume est très important. La chirurgie à ciel ouvert est nécessaire en cas de rupture urétérale importante [3].

\section{Références}

[1] Porfyris O, Apostolidi E, Mpampali A, Kalomoiris P. Spontaneous rupture of renalpelvis as a rare complication of ureteral lithiasis. Turk J Urol 2016;42:37-40.

[2] Garg PK, Mohanty D, Rathi V, Jain BK. Spontaneous rupture of the renal pelvispresenting as an urinoma in locally advanced rectal cancer. World J Clin Cases2014;2:108-10.

[3] Bogdanovic J, Djozic J, Idjuski S, Popov M, Sekulic V, Stojkov J. Successful surgicalreconstruction of ruptured renal pelvis following blunt abdominal trauma. Urollnt 2002;68:302-4.

[4] Bannowsky A. latrogenic fornix rupture caused during retrograde manipulationof the ureter: a case report. Cases J 2008;1:320.[5] Stathopoulos L, Merrot T, Chaumoitre K, Bretelle F, Alessandrini P. Prenatalurinoma related to ureteropelvic junction obstruction: poor prognosis of theaffected kidney. Urology 2010;76:190-4. 
Fig. 1. Vue coronale du scanner au temps veineux (a) montrant un épanchement liquidien périuretéral gauche (flèches). Au temps tardif, le scanner montre l'extravasationde produit de contraste au sein de l'épanchement liquidien péri-rénal et urétéral, confirmant l'origine urinaire de celui-ci (flèches) en coupe coronales (b) et axiales (c). Aucune obstruction urétérale n'était visualisée incluant l'uretère pelvien (flèches) et la vessie (d).

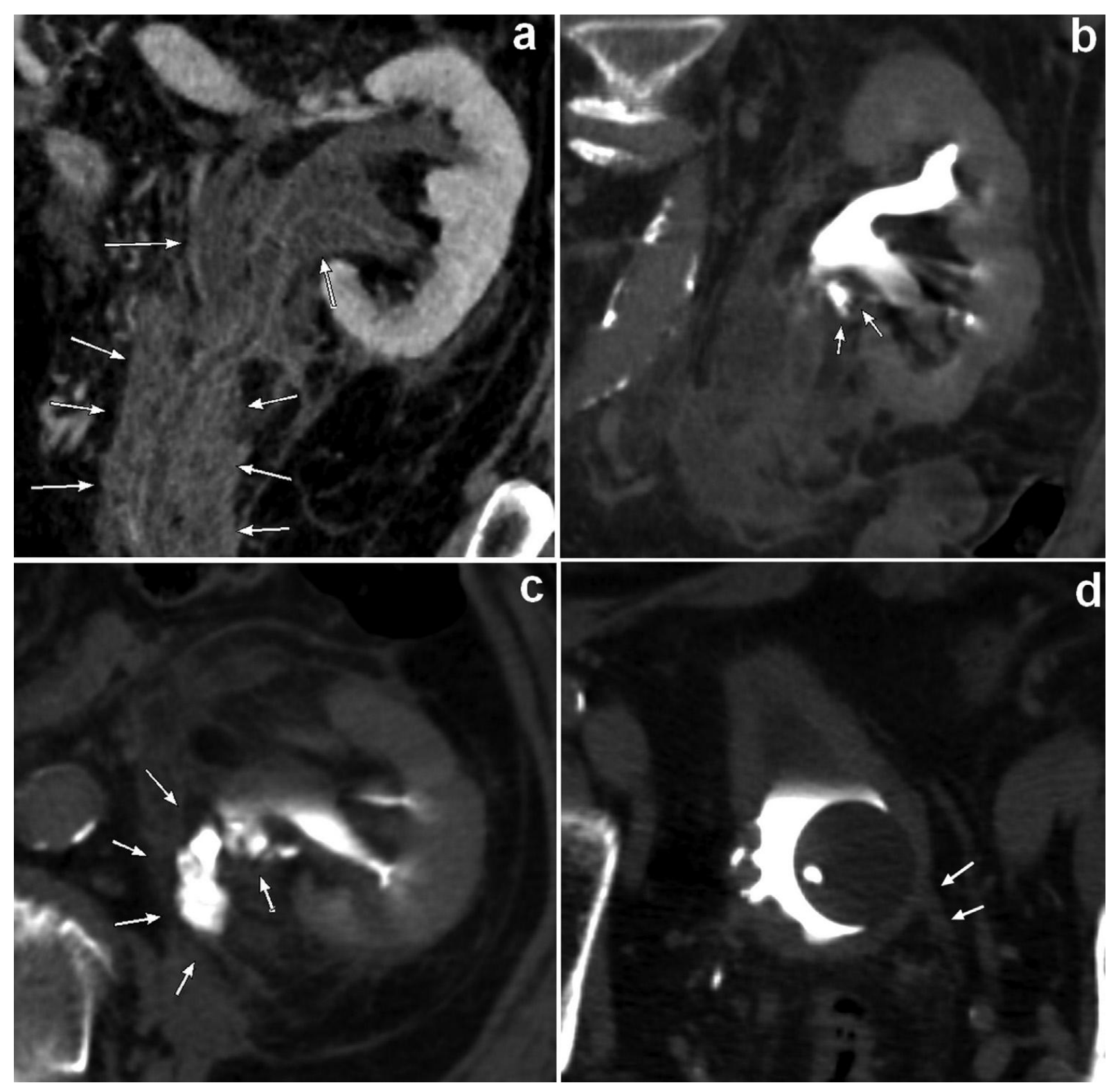

